\title{
Position accuracy and repeatability calibration of turn tables by using laser interferometers
}

\author{
I. Ahmet Yüksel ${ }^{1}$, T. Oytun Kılınç ${ }^{1}, K$. Berk Sönmez ${ }^{1}$, and Sinem Ön Aktan ${ }^{1}$ \\ ${ }^{1}$ Department of Laboratories and Calibration Management, Roketsan Missiles Industries Inc., 06780 \\ Ankara, Turkey
}

\begin{abstract}
This paper presents how to perform position accuracy calibration of multi axis turntable with a laser interferometer according to ISO-230-2 Determination of accuracy and repeatability of positioning of numerically controlled axes standard [1]. High accurate multi-axis turn tables are frequently used at defense and aviation \& space industries. These turn tables are used to control of devices which collect data regarding position. So turn tables shall be calibrated periodically to remain between specification performances criteria. Calibration of a rotary axis of turn tables' position is commonly performed by using autocollimator and multi-sided polygon systems. Alignment of polygon and autocollimator with turn table axes may have difficulties regarding time requirements and setup of hardware. Laser interferometers can be used as an alternative method to regular calibration of position and repeatability of an axis with advantages considering time requirement and ease of application. In this study, position accuracy and repeatability calibration of a horizontal axis which has \pm 3 arc second will be performed and results will be evaluated.
\end{abstract}

\section{Introduction}

Multi axis turn tables are generally used to control very critical devices which are used to send position information before final assembly and due to importance of test it shall be calibrated properly for better accuracy values during the ground test. A turn table may have accuracy problem due to wobble on axis, orthogonality and position accuracy. These errors give information regarding the operation of high precision radial encoders and servo motor. With current technology multi axis turn table position accuracy can be low as 1 arc-second which is very hard to check. By the help of perfect cooperation of encoder and servo-motor without any back leash affect, \pm 1 arc-second position accuracy can be achieved on both horizontally or vertically aligned axes.

Such accurate position accuracy shall be checked by very precision measurement systems. For this kind of applications, environmental conditions are also very effective while taking measurements. So in order to check and calibrate position accuracy of horizontal and vertical axes, very accurate system shall be installed and these measurement systems shall be operated under controlled environments to deduct errors other from turn-table.

\footnotetext{
* Corresponding Author : ahmet.yuksel@roketsan.com.tr
} 
Original Equipment Manufacturers are using high accurate auto-collimators, counters and polygons to calibrate position accuracy of inner and outer axes of turn tables (horizontal and vertical). As calibration methods, system is sent to several positions and by the help of autocollimators exact position information is collected. Generally position interval $45^{\circ}$ is selected for calibration. So comparing these two exact and nominal position values as per statistical approaches by ISO 230-2 standard [1], it is decided whether encoder and servo cooperation is satisfying or not for each axes. As an alternative way to autocollimator and polygon system, this measurement can be performed by using laser interferometer devices with same accuracy level. By using laser interferometers, especially for horizontal axes, some advantages can be achieved as they can be also used for calibration of motion systems including CMMs and machine tools. Also by the help of developed technology, laser interferometer based sensing and tracking system are started to be used for performing real time position measurements of dynamic systems such as robot manipulators[2]. Beside calibration purposes, laser interferometer systems had provided significant benefits in many manufacturing technologies and other areas [3].

So in this study, it is planned to perform a horizontal axis calibration of one high accurate multi axis turn table by using a laser interferometer system with all mathematical calculation philosophy. Furthermore position repeatability will be calculated on the basis of same method. Beside those, automatic calibration method of multi axis turn tables by communication ability of laser interferometers and control unit of device will be mentioned for easy and convenient applications.

\section{Calculations}

Position accuracy of linear axis and rotary axis are described at ISO-230-2 standard. Beginning of the calibration the environment conditions shall be satisfied as agreed with manufacturer. Ideally all dimensional measurement are made when the both the measurement instrument and measured object and room temperature at $20^{\circ} \mathrm{C}$. If these temperature different from the $20^{\circ} \mathrm{C}$, necessary correction has to applied yield result to $20^{\circ} \mathrm{C}$. So it is important to collect temperature data from machine to be calibrated and room in order to increase accuracy of measurement [1].

\subsection{Position accuracy and repeatability}

Minimum number of target point should be selected for rotary axis as per below table.

Table 1. Target positions for rotary axes [1].

\begin{tabular}{|c|c|}
\hline Measurement Travel & $\begin{array}{c}\text { Minimum number of target } \\
\text { positions }\end{array}$ \\
\hline$\leq 90^{\circ}$ & 3 \\
\hline $90^{\circ}<$ and $\leq 180^{\circ}$ & 5 \\
\hline$>180^{\circ}$ & 8 \\
\hline
\end{tabular}

According to ISO-230-2 below parameters should be checked by for the machine calibration and acceptance. These parameters have to be within tolerances specified by the manufacturer. Before verification of below parameters, regarding environmental conditions and devices which will be used for calibration should be discussed. Devices should have a valid calibration certificate and also should be capable to perform measurement considering accuracy details as per machine tolerance bandwidths. 
Table 2. Performance parameters [1]

\begin{tabular}{|c|c|}
\hline Parameters & Characteristic \\
\hline Bi-directional positioning error of an axis & $\mathrm{A}$ \\
\hline Unidirectional positioning error of an axis & $\mathrm{A} \uparrow$ and $\mathrm{A} \downarrow$ \\
\hline $\begin{array}{c}\text { Bi-directional systematic positioning error } \\
\text { of an axis }\end{array}$ & $\mathrm{E}$ \\
\hline $\begin{array}{c}\text { Unidirectional systematic positioning error } \\
\text { of an axis }\end{array}$ & $\mathrm{E} \uparrow$ and $\mathrm{E} \downarrow$ \\
\hline $\begin{array}{c}\text { Range of the mean bi-directional } \\
\text { positioning error of an axis }\end{array}$ & $\mathrm{M}$ \\
\hline $\begin{array}{c}\text { Bi-directional positioning repeatability of } \\
\text { an axis }\end{array}$ & $\mathrm{R} \uparrow$ and $\mathrm{R} \downarrow$ \\
\hline $\begin{array}{c}\text { Unidirectional positioning repeatability of } \\
\text { an axis }\end{array}$ & $\mathrm{B}$ \\
\hline Reversal error of an axis & $\mathrm{B}$ \\
\hline Mean reversal error of an axis
\end{tabular}

In order to determine accuracy of a rotary axis below calculations shall be executed by respectively as it is given at [1].

Positioning deviation (deviation of position);

$$
X i j=P i j-P i
$$

$\mathrm{P}_{\mathrm{i}}=$ Target position

$\mathrm{P}_{\mathrm{ij}}=$ Actual position

Mean unidirectional positioning deviation at a position;

$$
\begin{aligned}
& (X i) \uparrow=\frac{1}{n} \sum_{j \neq 1}^{n}(X i j) \uparrow \\
& (X i) \downarrow=\frac{1}{n} \sum_{j=1}(X i j) \downarrow
\end{aligned}
$$

Mean bi-directional positioning deviation at a position;

$$
\overline{X i}=\frac{(X i) \uparrow+(X i) \downarrow}{2}
$$

Reversal error at a position;

$$
B i=(X i) \uparrow-(X i) \downarrow
$$

Reversal Error of an axis;

$$
B=\max .[|\mathrm{Bi}|]
$$


Mean reversal error of an axis;

$$
(B i)=\frac{1}{m} \sum_{i=1}^{m}(B i)
$$

Estimator for the unidirectional axis positioning repeatability at a position;

$$
S i \uparrow=\sqrt{\frac{1}{n-1} \sum_{j=1}^{n}\left[\left(X_{i j}\right) \uparrow-\left(X_{i}\right) \uparrow\right]^{2}}
$$

and

$$
S i \downarrow=\sqrt{\frac{1}{n-1} \sum_{j=1}^{n}\left[\left(X_{i j}\right) \downarrow-\left(X_{i}\right) \downarrow\right]^{2}}
$$

Unidirectional positioning repeatability at a position;

$$
\begin{aligned}
& R i \uparrow=4 S i \uparrow \\
& R i \downarrow=4 S i \uparrow
\end{aligned}
$$

Bi-directional positioning repeatability at a position;

$$
R i=\max .[2 S i \uparrow+2 S i \downarrow|B i| ; R i \uparrow ; R i \downarrow]
$$

Unidirectional positioning repeatability of an axis;

$$
\begin{aligned}
& R \uparrow=\max \left[\begin{array}{ll}
R i & \uparrow
\end{array}\right] \\
& R \downarrow=\max \left[\begin{array}{ll}
R i & \downarrow
\end{array}\right]
\end{aligned}
$$

Bidirectional positioning repeatability of an axis;

$$
R=\max [R i]
$$

Unidirectional systematic positioning error of an axis;

$$
\begin{aligned}
& E \uparrow=\max .\left[\begin{array}{ll}
X i & \uparrow
\end{array}\right]-\min [X i \uparrow] \\
& E \downarrow=\max .[X i \downarrow]-\min [X i \downarrow]
\end{aligned}
$$

Bi-directional systematic positioning error of an axis;

$$
E=\max .[X i \uparrow ; X i \downarrow]-\min [X i \uparrow ; X i \downarrow]
$$

Mean bi-directional positioning error of an axis;

$$
\mathrm{M}=\max [\overline{X i}]-\min [\overline{\mathrm{Xi}}]
$$

Unidirectional positioning error of an axis;

$$
A \uparrow=\max .[\overline{\mathrm{Xi}} \uparrow+2 \mathrm{Si} \uparrow]-\min \overline{[\mathrm{Xi}} \uparrow-2 \mathrm{Si} \uparrow]
$$


Bi-directional positioning error of an axis;

$$
\mathrm{A}=\max [X i \uparrow+2 S i \uparrow ; X i \downarrow+2 S i \downarrow]]-\min [X i \uparrow-2 S i \uparrow ; X i \downarrow-2 S i \downarrow]
$$

\section{Application}

For measurements, Renishaw XL-80 laser interferometry systems and XR20 W Rotary Head are used with the program named Carto-Capture which is dedicated to be operated with Renishaw laser interferometry systems. Software is dedicated to collect data with Renishaw laser systems for linear and angular measurements.

XR20-W Rotary system is equipped with angular retroreflector which is mounted on servo-controlled axis which has high precision. Optics which are used to measure angular position of an axis is controlled by a high precision encoder.

Basically Renishaw Laser interferometer has a technology which is derived from The Michelson interferometry basic principle. As Michelson invented at 1880's, basic scale of the interferometer is the wavelength of source in the air. Red color laser sources which called as also He-Ne lasers are commonly used as a source because of proper length, power level and the high state of development of this laser [4]. Accuracy of optical interferometry had been dramatically increased after invention of lasers.

In detailed, by using two light beams which are divided by a splitter from one beam, an interference pattern can be formed when these two beams superpose. Because the wavelength of the visible light is very short, small changes in the differences in the optical paths (distance travelled) between the two beams can be detected [5].

The linear measurement principle of laser interferometer which will be used for measurement of a linear axis as shown below Fig. 1 .

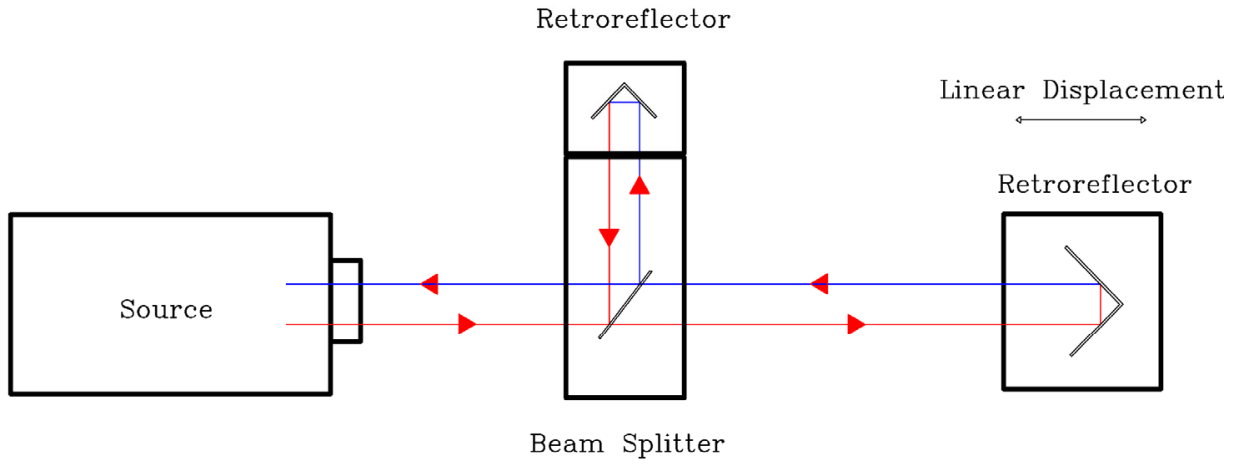

Fig 1. Laser interferometry basic schematic.

As it is also illustrated at Figure 1, movement of retroreflector causes change in phase of both incoming and returning beams. During movement, at every motion which equals to half wavelength of source, change of brightness of source to be returned is counted as a cycle.

Exact movement information can be determined by below equation. Better accuracies can be derived by applying interpolation between cycles.

$$
d=\frac{\lambda N}{2}
$$

An example setup with laser source located left side, beam splitter located into middle as perpendicular to beam from laser source and rotary head on the machine axis on the right are illustrated at Fig.2. 


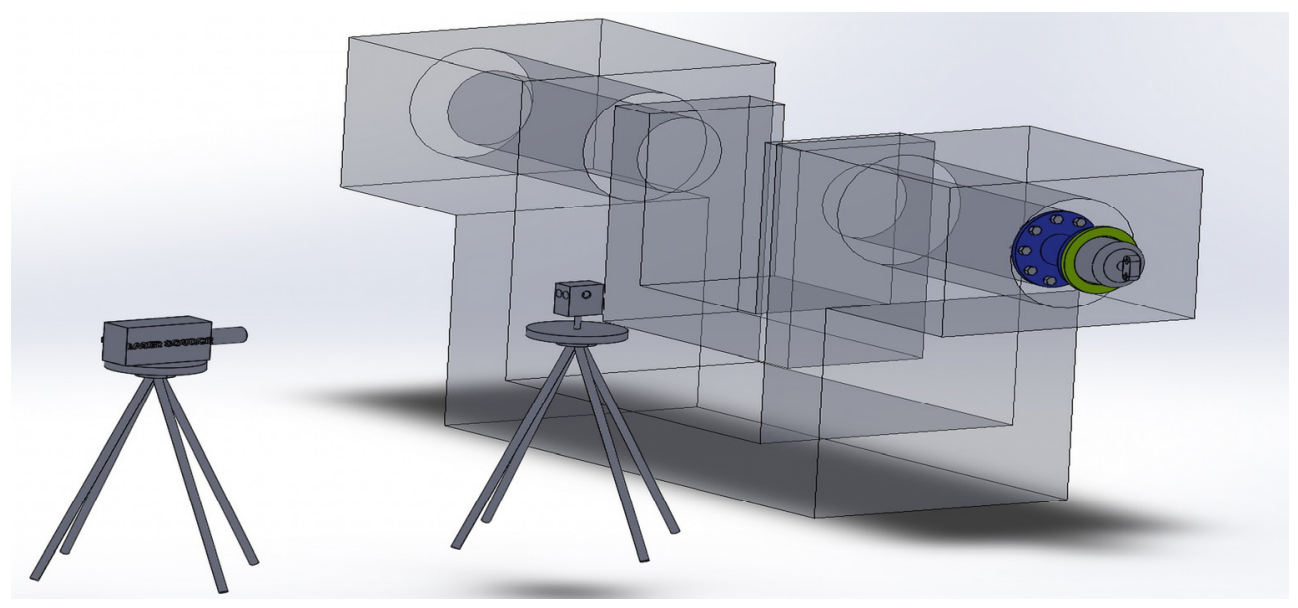

Fig.2. Laser interferometer measurement system illustration.

Accuracy of Renishaw XL- 80 laser is given as $\pm 0.5 \mathrm{ppm}$ for linear measurements. This level of accuracy is valid at accurate environmental compensation. Linear Resolution of device is given as 1 nanometers $(\mathrm{nm})$. Accuracy for rotary measurements at same conditions is given as down to \pm 1 arc-second and this angular accuracy is equivalent of approximately 5 microns movement at 1 meter.

After setting the laser source, splitter and rotary head which is aligned according to axis of turn table with mechanical adapter as discussed at section 3.1, test plan should be created over software. Program shall be created by considering target points, over-run for back leash, data collections details such as residence time, averaging methods.

Minimum 8 points have to be chosen as target in both directions and should be measured five times. The target positions have been shown below table. For the eliminate the back leash effect the test start with $5^{\circ}$ overrun in each round and each direction. In case of back leash problem, it is expected to see over deviations from actual position at $0^{\circ}-360^{\circ}$ positions before after changing direction of movement. Sequence of movement is adjusted as bilateral.

Table 3. Selected Target Positions

\begin{tabular}{|c|c|}
\hline Target Point & Degree \\
\hline 0 & $0^{\circ}$ \\
\hline 1 & $45^{\circ}$ \\
\hline 2 & $90^{\circ}$ \\
\hline 3 & $135^{\circ}$ \\
\hline 4 & $180^{\circ}$ \\
\hline 5 & $225^{\circ}$ \\
\hline 6 & $270^{\circ}$ \\
\hline 7 & $315^{\circ}$ \\
\hline 8 & $0^{\circ}$ \\
\hline
\end{tabular}

While measurements, by the help of auto-detection system of XR20-W rotary head, speed of axis rotation can be recognized automatically within the range of rotary head speed limits. However it is also possible to set speed parameter before measurement as it can be also set on turn table. 
In this study, rotation speed is selected and set as $10 \%$ second before measurement at both measurement system and turn table control system, device capacity is declared as 10 revolution per minute (rpm) which is equal to $60 \%$ second.

Since turn table is controlled by a PLC, test destinations and residance time at each target can be set. After running test, all measurement can be taken without any operator intervention. For each target position, approximately 10 seconds stabilization time are set to collect data. When considering totally 80 targets, all test can be performed within 15 minutes after obtaining orientation of laser and device excluding the travel time which is elapsed while moving from one position to another position.

\subsection{Mechanical adaptor between turn table horizontal axis and rotary head mounting ring}

Measurement of horizontal axis of turn tables is relateviley harder when comparing with vertical ones due to mounting problem of rotary head which transfer precise angular position information by using laser beam and its retroreflector which is equipped with encoder-servo system.

Generally for turn tables with air conditioning systems, horizontal axis of turn tables are coupled with ventilation hoses in order to obtain desired temperature inside of cabinet where product is tested under various temperatures. So before position accuracy tests, air conditioning system system shall be dismounted carefully.After dismounting of outer axis casing and other auxilarry equipment, rotary head shall be alligned to horizantal axis with minimum requirements as it is declared by manufacturer to obtained best accuracy of measurement by mean of rotary ring and an adapter.

So, a mechanical adapter between interface of turn table axis outlet and rotary head's mounting ring shall be designed and manufactured with below geometrical and dimensional requirements. Adapter shall be periodically calibrated in order to ensure that geometrical features and dimension remain same.

Before manufacturing adapter, firstly, interface of rotary axis outlet where adapter will be mounted, shall be measured to design adapter's connection details. Threaded connection details shall be known and applied to adapter surface for easy mounting. Furthermore, adapter should not be manufactured as single body due to rework possibility. In case adapter geometry is out of tolerance limits, by the help of threaded connection of cylinder which is shown by Fig.3. rework process can be applied until desired conditions are maintained again.

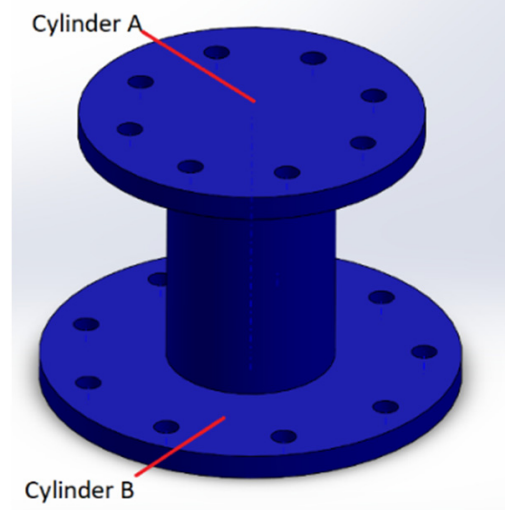

Fig 3. Adapter between mounting ring and rotary axis

As it is shown at Fig. 3, cylinder A top surface, cylinder B bottom surface shall be parallel to each other with maximum $0.03 \mathrm{~mm}$ in order to keep calibrator axis and rotary axis 
coincident. Also coaxility of both cylinders should be maximum $0,05 \mathrm{~mm}$ to keep axis of rotary head and axis of device parallel within limits of manufacturer which is mentioned below.

After succesfull setup of hardwares, laser beam quality should be checked to ensure high level of connection. Quality of created beam can be observed by checking indication system on laser source. Created laser beam quality also affects accuracy of system.

In order to eliminate setup errors, mounting of rotary head and adapter shall be performed according to manufacturer declaration related to centration of rotary head to machine axis. Centration of mounting ring of rotary head axis of turn table should be lower than $\pm 1 \mathrm{~mm}$ by using a dial indicator. Even if the centration is performed succesfull, also axis of rotary head and turn table axis should be paralel to each other. Deviation on axes parallesim affect the measurement results and creates induced error which can be cseen on results as sinusodial. So, for induced error to be less than \pm 1 arcsecond, ensure paralelism error is lower than $0.025^{\circ}$ or the total indication run out is lower than $0.04 \mathrm{~mm}$ at a radius of 50 $\mathrm{mm}$ from the centre of rotation of the axes. Also laser beam should be perpendicular to reflector with a maximum 2 mrad deviation.[5]

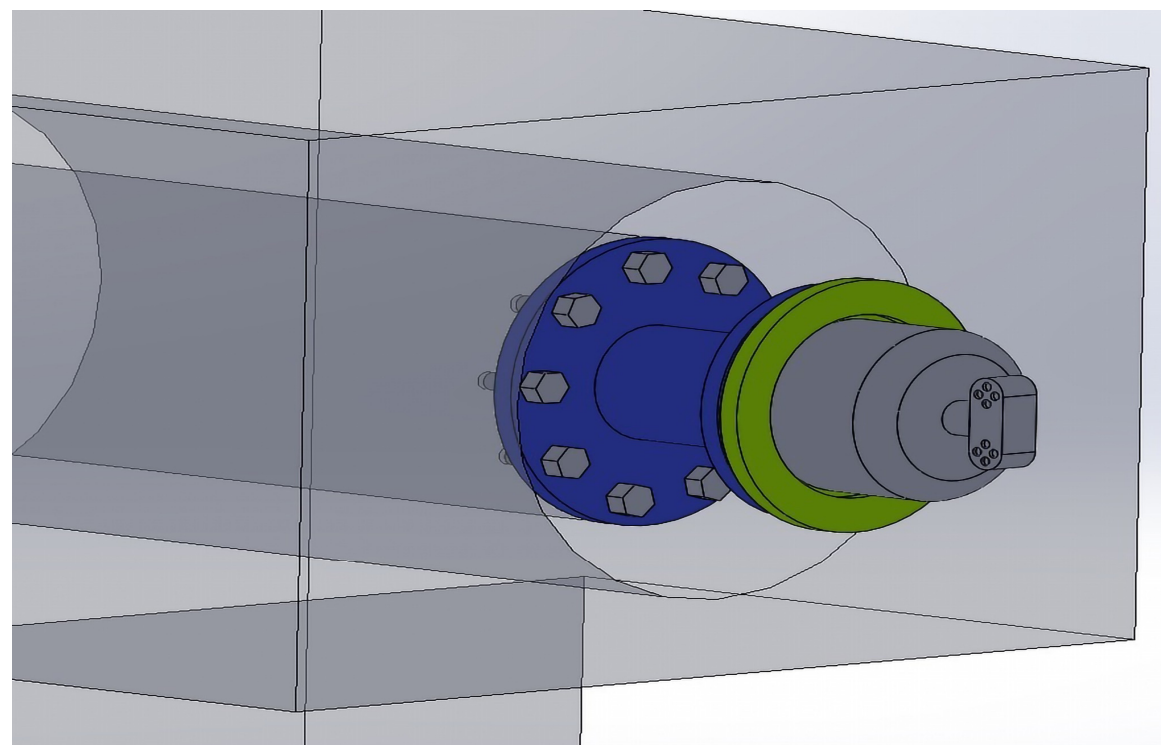

Fig 4. Adapter and XR20-W on an horizantal axis and adapter

\section{Results and conclusion}

In this study, horizontal axis of two axis turn table with \pm 3 arc-second accuracy is tested. After collecting data from each target for both directions 5 times, analyses had been performed as per ISO 230-2 requirements which explained in detailed at section 2. Deviations of each position had been calculated as per equation (1) and further calculation are executed in order to determine position accuracy and repeatability of each axes. Results are processed and graphics are prepared by using Carto Explore 3.0. 
Table 4. Results of horizontal axis with \pm 3 arc second position accuracy of targets 1 to 4 .

\begin{tabular}{|c|c|c|c|c|c|c|c|c|c|}
\hline \multicolumn{10}{|c|}{ Results Between $0^{\circ}$ to $135^{\circ}$} \\
\hline Target no (i) & $\begin{array}{c}1 \\
(-)\end{array}$ & $\begin{array}{c}1 \\
(+)\end{array}$ & $\begin{array}{c}2 \\
(-)\end{array}$ & $\begin{array}{c}2 \\
(+)\end{array}$ & $\begin{array}{c}3 \\
(-)\end{array}$ & $\begin{array}{c}3 \\
(+)\end{array}$ & $\begin{array}{c}4 \\
(-)\end{array}$ & $\begin{array}{c}4 \\
(+)\end{array}$ & $\begin{array}{c}5 \\
(-)\end{array}$ \\
\hline Position $\left({ }^{\circ}\right)$ & 0 & 0 & 45 & 45 & 90 & 90 & 135 & 135 & 180 \\
\hline Direction & - & + & - & + & - & + & - & + & - \\
\hline $\mathrm{Xij} \mid \mathrm{j}=1$ & 0.13 & 0.00 & 1.04 & 0.50 & 0.29 & 0.15 & -0.51 & -1.12 & $-0,60$ \\
\hline $\mathrm{Xij} \mid \mathrm{j}=2$ & 0.10 & 0.07 & 0.80 & 0.84 & 0.30 & 0.30 & -0.85 & -0.66 & $-0,32$ \\
\hline $\mathrm{Xij} \mid \mathrm{j}=3$ & 0.32 & 0.14 & 0.86 & 0.98 & 0.41 & 0.52 & -0.47 & -0.38 & $-0,39$ \\
\hline $\mathrm{Xij} \mid \mathrm{j}=4$ & 0.41 & 0.19 & 1.05 & 0.89 & 0.28 & 0.83 & -0.57 & -0.40 & $-0,42$ \\
\hline $\mathrm{Xij} \mid \mathrm{j}=5$ & 0.34 & 0.27 & 1.04 & 0.98 & 0.64 & 0.78 & -0.29 & 0.10 & $-0,59$ \\
\hline Mean deviations & 0.26 & 0.14 & 0.96 & 0.84 & 0.39 & 0.51 & -0.54 & -0.49 & $-0,46$ \\
\hline Standard uncertainty & 0.13 & 0.11 & 0.12 & 0.20 & 0.15 & 0.30 & 0.20 & 0.45 & $+0,13$ \\
\hline $\mathrm{Si}$ & &
\end{tabular}

Table 5. Results of horizontal axis with \pm 3 arc second position accuracy of targets 6 to 9 .

\begin{tabular}{|c|c|c|c|c|c|c|c|c|c|}
\hline \multicolumn{10}{|c|}{ Results Between $180^{\circ}$ to $360^{\circ}$} \\
\hline Target no (i) & $\begin{array}{c}5 \\
(+)\end{array}$ & $\begin{array}{c}6 \\
(-)\end{array}$ & $\begin{array}{c}6 \\
(+)\end{array}$ & $\begin{array}{c}7 \\
(-)\end{array}$ & $\begin{array}{c}7 \\
(+)\end{array}$ & $\begin{array}{c}8 \\
(-)\end{array}$ & $\begin{array}{c}8 \\
(+)\end{array}$ & $\begin{array}{c}9 \\
(-)\end{array}$ & $\begin{array}{c}9 \\
(+)\end{array}$ \\
\hline Position $\left({ }^{\circ}\right)$ & 180 & 225 & 225 & 270 & 270 & 315 & 315 & 360 & 360 \\
\hline Direction & + & - & + & - & + & - & + & - & + \\
\hline $\mathrm{Xij} \mid \mathrm{j}=1$ & -0.67 & 0.13 & 0.00 & -0.24 & -0.16 & -0.26 & 0.01 & 0.10 & -0.08 \\
\hline $\mathrm{Xij} \mid \mathrm{j}=2$ & -0.24 & 0.41 & 0.53 & 0.06 & 0.07 & -0.32 & -0.15 & - & -0.01 \\
\hline $\mathrm{Xij} \mid \mathrm{j}=3$ & -0.10 & 0.63 & 0.50 & 0.09 & -0.07 & -0.23 & 0.20 & - & 0.11 \\
\hline $\mathrm{Xij} \mid \mathrm{j}=4$ & -0.36 & 0.44 & 0.73 & 0.30 & 0.18 & -0.08 & -0.37 & - & 0.02 \\
\hline $\mathrm{Xij} \mid \mathrm{j}=5$ & -0.14 & 0.69 & 0.88 & 0.52 & 0.34 & 0.29 & 0.20 & 0.25 & 0.28 \\
\hline Mean deviations & -0.30 & 0.46 & 0.53 & 0.14 & 0.07 & -0.12 & -0.02 & 0.04 & 0.09 \\
\hline $\begin{array}{c}\text { Standard } \\
\text { uncertainty Si }\end{array}$ & -0.23 & 0.22 & 0.33 & 0.28 & 0.20 & 0.25 & 0.24 & 0.14 & 0.25 \\
\hline
\end{tabular}

Table 6. Summary of results as per ISO 230-2 Standard to evaluate machine.

\begin{tabular}{|c|c|c|c|}
\hline Name & $(+) \operatorname{arcsec}$ & $(-) \operatorname{arcsec}$ & (Bildir) arcsecs \\
\hline Positioning error (A) & 2,62 & 2,14 & 2,62 \\
\hline Positioning repeatability (R) & 1,79 & 1,13 & 1,79 \\
\hline Systematic positioning error (E) & 1,33 & 1,50 & 1,50 \\
\hline Reversal (B) & & & 0,16 \\
\hline Mean reversal & & & 0,03 \\
\hline $\begin{array}{c}\text { Range of mean bidirectional positioning error } \\
(M)\end{array}$ & & & 1,42 \\
\hline
\end{tabular}

After obtaining results, it has been seen that results of original equipment manufacturer by using autocollimator system and 8 sided polygon is very similar with results obtained by laser interferometer system. From the view of ease of application and time requirement, 
optical interferometry can be considered as an alternative method for position accuracy and repeatability calibration of turn tables for their both horizontal and vertical axes.

Source of errors of a rotary axis accuracy can be categorized at 3 main items. These three main sources cover following errors respectively, geometric inaccuracies, thermally induced errors and load induced errors [6]. For elimination of these affect or keeping them at minimum level, required action shall be obtained. Thermal sensors should be used for during measurement for calculation of thermal uncertainty affects and inertial scaling adjustment of turn table should be done by using counterweights before measurement.

Regarding uncertainty approach for position accuracy measurements, below contributors are mentioned at ISO 230-2. These sources should be taken in to consideration while calculations.

- Uncertainty due to measuring device

- Uncertainty due to misalignment of measuring device to rotary axis

- Uncertainty due to machine temperature compensation

- Uncertainty due to environmental variation error

In case of evaluation of results, if results are not satisfying, a correction can be implemented electronically by the help of obtained deviations for each steps. In some cases, also mechanical adjustments may be needed. After all corrections, all test procedure shall be repeated. Also, wobble and orthogonality of turn table should be controlled and calibrated for reliable usage.

Further study may cover uncertainty calculations of position accuracy measurements of rotary axis by using laser interferometers with a mechanical connection adapter to machine axis.

İhsan Ahmet YÜKSEL acknowledges the support of Roketsan Missiles Industries Inc.

\section{References}

1. International Organization for Standardization, ISO 230-2 - Determination of accuracy and repeatability of positioning of numerically controlled axes, (2014)

2. P.L. Teoh, B. Shirinzadeh, C.W. Foong, G. Alici, The measurement uncertainties in the laser interferometry-based sensing and tracking technique, Measurement 32, 2, 135-150, (2002)

3. C.R. Steinmetz, Sub-micron position measurement and control on precision machine tools with laser interferometry, Precision engineering 12, 1, 12-24 (1990)

4. N. Bobroff, Residual errors in laser interferometry from air turbulence and nonlinearity, Applied optics 26, 13, 2676-2682, (1987)

5. Renishaw User Guide, XR20-W Rotary Axis Calibrator, F-9950-0400-01 A (2018)

6. A.C. Okafor, and Y.M. Ertekin, Vertical machining center accuracy characterization using laser interferometer: part 1. Linear positional errors, Journal of Materials Processing Technology, 105, 3, 394-406 (2000) 